\title{
Políticas de acesso universal à banda larga: propostas para o Brasil
}

\author{
André Moura Gomes, Pedro Antero Braga Cordeiro, Pedro lucas da Cruz Pereira Araúuo
}

\begin{abstract}
Resumo
O objetivo da pesquisa é apresentar propostas de políticas de acesso universal à Internet em banda larga, avaliando a hipotética aplicação ao Brasil de medidas sugeridas pela literatura setorial. A metodologia empregada se baseia na análise da literatura a respeito de políticas de acesso universal e planos de banda larga no âmbito dos países da América Latina e da Organização para a Cooperação e Desenvolvimento Econômico (OCDE), bem como na discussão do seu efeito sobre os indicadores setoriais relacionados. Em seguida, estima-se o impacto da aplicação de três abordagens de políticas de acesso universal sobre indicadores de acesso à banda larga no Brasil: estímulo à oferta de acesso por meio de destinação de recursos para implantação de infraestrutura em regiões carentes de atendimento, estímulo à demanda utilizando subsídios diretos ao consumo e desoneração tributária do serviço. Por fim, são comparados os resultados esperados de cada politica e se sugere um modelo hibrido com o propósito de maximizar o benefício social de cada abordagem.
\end{abstract}

Palavras-chave: Banda larga, Política Pública, Universalização, Brasil. 


\section{Introdução}

O objetivo da pesquisa é apresentar propostas de políticas de acesso universal à Internet em banda larga, avaliando medidas sugeridas pela literatura setorial e os impactos da sua aplicação hipotética ao Brasil. Embora haja estudos que abordam a relação entre desenvolvimento e serviços de telecomunicações desde a década de 1980 (Hardy, 1980), recentemente têm ganhado destaque na literatura os estudos que mensuram o impacto do acesso à banda larga no Produto Interno Bruto - PIB dos países, ou no desenvolvimento socioeconômico em sentido amplo (Qiang e Rossotto, 2009; Koutrompis, 2009; COMISSÃO EUROPEIA, 2012).

Paralelamente, proliferam no mundo conjuntos de ações governamentais voltados à expansão de infraestrutura de alta capacidade de tráfego de dados e do acesso à banda larga em geral, inclusive na América Latina e Europa (Galperín et al., 2012; Calvo, A.G., 2012). Boa parte das ações governamentais previstas nos diversos planos se fundamenta numa análise dos principais gargalos encontrados em cada região e nas recomendações de política pública discutidas globalmente. Neste artigo, traça-se um panorama das principais recomendações de política pública encontradas na literatura e, em seguida, estima-se o impacto da aplicação de três instrumentos selecionados ao caso brasileiro: estímulo à oferta de acesso à banda larga por meio de destinação de recursos para implantação de infraestrutura em regiões mal atendidas, estímulo à demanda utilizando subsídios diretos ao consumo e redução de tributos sobre o serviço. Por meio da avaliação dos impactos da sua aplicação hipotética ao Brasil, postula-se a possibilidade de propor um modelo híbrido de utilização dos instrumentos que otimize a aplicação de recursos, em face das peculiaridades de cada um dos instrumentos.

0 trabalho está organizado da seguinte maneira. Na primeira seção, passa-se à análise resumida da literatura a respeito de políticas de acesso universal e planos de banda larga no âmbito dos países da América Latina e da Organização para a Cooperação e Desenvolvimento Econômico (OCDE). A discussão está centrada na identificação das políticas mais aderentes ao contexto socioeconômico de países emergentes.

Em seguida, são descritos os instrumentos selecionados, suas vantagens e desvantagens, os parâmetros utilizados no respectivo modelo econômico e os resultados de sua aplicação hipotética ao Brasil. De um lado, a destinação de recursos para estímulo à oferta de infraestrutura e serviços de telecomunicações em regiões mal atendidas tende a gerar impacto localizado, em função da limitação territorial da medida. Por outro lado, este instrumento pode ser o mais indicado em projetos não economicamente viáveis no médio prazo para implantar infraestrutura em regiões isoladas ou incentivar a implantação de infraestrutura de telecomunicações com uma tecnologia específica. Para além da expansão dos acessos, o crescimento projetado para o tráfego de dados nos próximos anos e a exigência de qualidade na prestação do serviço podem justificar o incentivo à adoção de tecnologias mais robustas. 
De outro lado, pode haver vantagens em concentrar os esforços de política pública em incentivos à demanda pelo serviço. Políticas de incentivo a conteúdos digitais e aplicações, vídeo sob demanda, jogos eletrônicos, governo eletrônico e capacitação de cidadãos e empresas são exemplos de algumas políticas dessa natureza (COMISSÃO DE COMÉRCIO, 2012). Neste trabalho se utiliza como referência de incentivo à demanda o modelo de subsídio direto ao consumo do serviço de banda larga, adotado em países como a Colômbia para extratos da população mais pobres (Vive Digital, 2011). Em princípio, este modelo pode ser indicado para áreas não economicamente viáveis no longo prazo (ou seja, com características socioeconômicas que não permitem ao agente privado avaliar que é possível atingir o rendimento esperado para o seu investimento, ainda que considerado um horizonte de tempo maior do que o adotado pelo mercado em geral).

A redução de tributos tende a gerar um impacto mais difuso no país. Entre as vantagens desta medida, podem ser citadas a sua abrangência territorial ${ }^{1}$, a facilidade de implementação e o seu impacto especialmente benéfico para a população de baixa renda. Para o mesmo pacote de serviço, o impacto da redução de tributos tende a ser maior para a população de baixa renda, pois o tributo, assim como o preço do serviço, corresponde a uma parcela maior de sua renda. A relação entre o preço do serviço e a renda da população é um dos principais indicadores correlacionados à alta penetração do serviço de banda larga (CEPAL, 2012). No entanto, a medida não garante que haverá redução do preço ao consumidor ou expansão da oferta do serviço para áreas mal atendidas, embora esses efeitos sejam esperados em mercados com algum grau de competição.

A conclusão do trabalho compara os resultados da aplicação hipotética dos três instrumentos acima e propõe um modelo híbrido de aplicação, considerando as peculiaridades de cada um.

\section{Revisão de literatura}

0 desenvolvimento associado à expansão do acesso à banda larga tem sido objeto de diversos estudos e relatórios, em especial desde 2009. Após análise de mais de 200 trabalhos, estudo da Analysys Mason e Tech4i2 (COMISSÃO EUROPEIA, 2012) aponta nove áreas em que os impactos podem ser destacados, conforme ressaltado no conjunto dos estados. Da lista apresentada, destaca-se a variedade de benefícios sociais para além do benefício econômico, que vão desde um incremento na participação social e política de comunidades locais até benefícios para a segurança pública, educação, meio ambiente e emprego.

1 Com exceção dos casos em que cabe à autoridade local conceder a isenção ou são criadas condições especiais para fruição da isenção, em princípio a isenção de tributos se aplica a todo o pais e a todas as faixas de preço de determinado serviço. 
Os efeitos mensurados variam de acordo com a metodologia aplicada e o objeto da análise. Ao que parece, no entanto, para um dado aumento na penetração de banda larga, o impacto no PIB é maior conforme o nível atual de uso da banda larga no país (CEPAL, 2012). Para um país com baixo número de acessos, o benefício marginal aumenta conforme o uso da banda larga se difunde em sociedade.

Com o objetivo de se inserir na sociedade da informação mais rapidamente, os diversos países têm se organizado internamente com o objetivo de implantar políticas de banda larga estruturadas, os chamados planos nacionais de banda larga. A comparação entre a evolução recente dos acessos à banda larga (ITU, 2012) e a existência de um plano nacional de banda larga nos diversos paises (Galperín et al., 2012; Calvo, A.G., 2012) permite identificar uma correlação positiva entre a adoção de planos de banda larga e evolução recente dos níveis de acesso.

Entre as políticas destacadas, estão políticas de investimento público direto (por exemplo, por meio do uso de fundos de universalização), incentivo à competição, redução de tributos, desenvolvimento de conteúdos digitais para fomentar a demanda, a disponibilização de espectro de radiofrequência em regime de utilização flexível, troca de experiências de melhores práticas com outros países, entre outras (Jordán, V., Galperin H. e Peres, W., 2010). De maneira geral, para cada política se pode esperar um impacto concentrado em um indicador específico.

Na análise agregada dos conjuntos de ações previstos nos diversos planos, é possível perceber uma distinção de foco entre os planos de países desenvolvidos e aqueles de países emergentes. Estabelecidos em contextos com altos índices de acesso, os planos nacionais de banda larga dos países da OCDE possuem foco em políticas de universalização para áreas rurais ou remotas e aumento da velocidade média. Além disso, apresentam uma ênfase relativamente maior em políticas de investimento em conteúdos digitais e aplicações, consideradas um importante fator gerador de demanda por infraestrutura de transporte de alta capacidade.

Em outro contexto político e socioeconômico, países considerados emergentes (incluindo os países da América Latina) tendem a concentrar seus esforços em infraestrutura e serviços de acesso à banda larga de caráter básico, de menor velocidade. Além do investimento em infraestrutura em regiões isoladas ou mal atendidas, também são indicadas políticas de desoneração tributária (ou redução de tributos) do serviço de acesso à banda larga, considerando a alta carga tributária média dos países emergentes. Por outro lado, a baixa renda per capita média desses países tem incentivado iniciativas de subsídio direto ao consumo do serviço pela população, como no caso da Colômbia (Vive Digital, 2011), Guatemala e Quênia. A depender do nível educacional da população, ocorrem também políticas de capacitação tecnológica básica.

Com o objetivo de otimizar recursos, o investimento em infraestrutura em regiões isoladas tem sido implementado por meio de política que se pode intitular de "leilão reverso". Trata-se de uma chamada pública com o objetivo de oferecer a menor quantidade de recursos 
públicos para cumprimento de dadas obrigações estabelecidas pela autoridade estatal. Em um processo de competição por recursos públicos, o setor privado assume o papel de definir qual é a parcela máxima do investimento total que está disposto a despender e sobra apenas a parcela do investimento não recuperável pelo negócio para ser subsidiada pelo Estado. Modalidades de tal política têm sido adotadas, por exemplo, no Chile (Proyecto Todo Chile Conectado, 2010) e nos EUA, com a utilização do Universal Service Fund (USF). Mais detaIhes sobre esse modelo de incentivo à infraestrutura e sobre as políticas de subsídio direto ao consumo e desoneração tributária serão vistos com mais detalhes na seção seguinte.

\section{Quatro propostas de política pública e seus impactos estimados para o Brasil}

Conforme visto na seção anterior, a experiência internacional e a literatura especializada recomendam diversos tipos de políticas públicas com o objetivo de incentivar a adoção de banda larga em todo o mundo. Desses instrumentos, foram selecionados três tipos de politicas que parecem mais adequadas para enfrentar os desafios presentes em países emergentes: a alta carga tributária, a carência de infraestrutura e a baixíssima propensão a pagar encontrada nas regiões mais pobres.

Após explanação sobre a metodologia de comparação entre as propostas de política pública, serão descritos os parâmetros e os resultados de cada uma (redução de tributos sobre o serviço, subsídio direto ao consumo do serviço e incentivos para investimento em infraestrutura). Na sequência, será apresentada a combinação entre os modelos (modelo híbrido).

\section{METODOLOGIA}

A análise dos resultados de cada proposta será conduzida com base em duas variáveis: a penetração percentual dos acessos fixos (definida como a razão entre o número total de acessos fixos e o número de domicílios) e a velocidade média desses acessos.

Cada uma das propostas exploradas oferece um resultado distinto para essas variáveis. Portanto, foi necessário definir um grau de valoração para cada um desses indicadores. Isso foi feito por intermédio de uma função que visa medir qual o impacto geral de cada política quando variamos esses dois parâmetros. Considerando não só a necessidade de se priorizar essas duas frentes em alguma medida, mas também a própria dependência entre as duas variáveis, a função F (ou "função Objetivo") foi definida em um formato côncavo do tipo:

$$
F(A, V)=A^{a} V^{v}
$$


Onde A e V são os indicadores dos níveis de penetração e velocidade média, respectivamente. Os coeficientes $a$ e $v$ foram estimados com base nos pesos assumidos por cada uma dessas variáveis no ICT Development Index - IDI da União Internacional de Telecomunicações (ITU, 2012).

Cabe ressaltar que, nos últimos anos, índices que visam medir a incorporação de tecnologias de informação e comunicação vêm exibindo um aumento relativo no peso da velocidade média em relação aos indicadores de penetração e acesso. Na composição dos índices, esse fato tem se revelado na evolução dos valores de referência. No cálculo do Networked Readiness Index, do Fórum Econômico Mundial (WEF, 2013), por exemplo, o valor de referência para a banda internacional por usuário subiu 147\% de 2012 para 2013. No mesmo período, o valor de referência para indicadores relacionados à penetração (como o número de domicílios com acesso à Internet e número de domicílios com acesso à banda larga fixa) não sofreu alterações significativas.

Para o caso do IDI, o valor base para a banda internacional evoluiu de 100.000 bits/s para 408.813 bits/s entre 2010 e 2012. Essas variações foram projetadas para estimar 0 formato da função $\mathrm{F}$ ao longo do tempo, como ilustrado na Figura 1.

Figura 1. Função Objetivo.
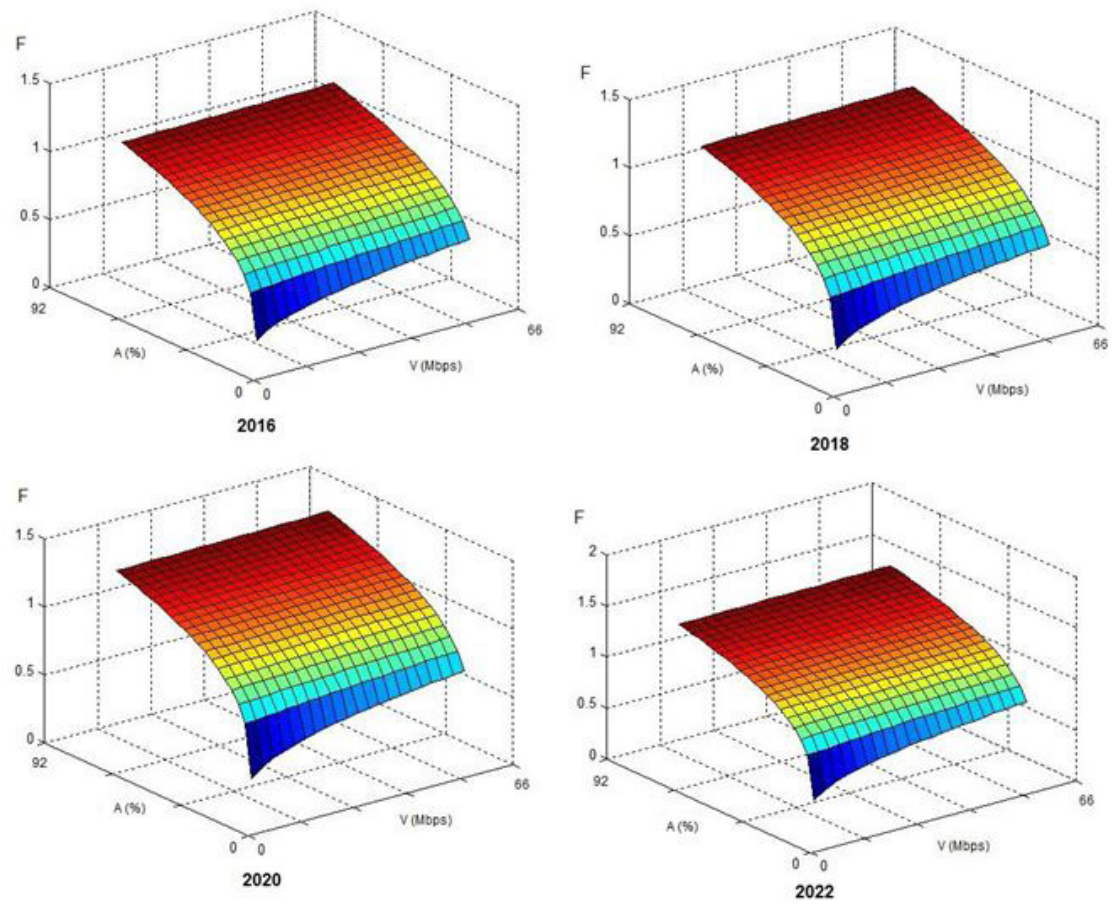
Ainda para viabilizar a comparação de cada um dos programas, foi definido o mesmo valor para o aporte governamental, estabelecido arbitrariamente como US\$ 20 bilhões (vinte bilhões de dólares) em valor presente no ano de 2013.

Além dessa restrição financeira, cada um dos modelos estudados possui limitações políticas inerentes. Nesse sentido, para efeitos desse estudo, será considerada bem sucedida a proposta que maximizar a função referida acima respeitando as restrições financeiras e políticas.

\section{MODELO 1 - DESONERAÇÃO TRIBUTÁRIA}

Nesta seção, tratamos da primeira opção de política pública voltada à difusão do acesso domiciliar à Internet em banda larga fixa no Brasil, que consiste na desoneração tributária da prestação desse serviço com o objetivo de reduzir o preço cobrado do usuário final. Começamos por introduzir a proposta de desoneração e, em seguida, apresentamos detalhadamente o método utilizado para avaliar seu impacto, baseado no cálculo de preços médios a partir de dados fiscais e na estimação de funções de demanda por Internet em banda larga fixa por meio da aplicação de técnicas de regressão a indicadores derivados de pesquisas domiciliares.

Os tributos que incidem diretamente sobre a fatura do serviço de acesso à Internet em banda larga fixa são apresentados no Quadro 1.

Quadro 1. Tributos incidentes sobre a fatura do serviço de acesso à Internet em banda larga fixa.

\begin{tabular}{|c|c|c|c|}
\hline Tributo & Descrição & Base de incidência & Alíquota \\
\hline ICMS & $\begin{array}{l}\text { Imposto sobre Operações relativas à Circulação de } \\
\text { Mercadorias e Prestação de Serviços de Transporte } \\
\text { Interestadual e Intermunicipale de Comunicação } \\
\text { Tributo que financia o Programa de Integração }\end{array}$ & Valor da fatura & $25 \%$ a $35 \%$ \\
\hline PIS & $\begin{array}{l}\text { Tributo que financia o Programa de Integração } \\
\text { Social }\end{array}$ & Valor da fatura & $3 \%$ \\
\hline Cofins & $\begin{array}{l}\text { Contribuição para Financiamento da Seguridade } \\
\text { Social }\end{array}$ & Valor da fatura & $0,65 \%$ \\
\hline Fust & $\begin{array}{l}\text { Tributo que compõe o Fundo de Universalização } \\
\text { dos Serviços de Telecomunicações } \\
\text { Tributo que compõe o Fundo para o }\end{array}$ & $\begin{array}{l}\text { Valor da fatura deduzido } \\
\text { de ICMS, PIS e Cofins } \\
\text { Valor da fatura deduzido }\end{array}$ & $1 \%$ \\
\hline Funttel & $\begin{array}{l}\text { Desenvolvimento } \\
\text { Telecomunicações }\end{array}$ & & $0,5 \%$ \\
\hline
\end{tabular}

A proposta de desoneração, que entraria em vigor em 2013, significaria a redução a zero das alíquotas do PIS, da Cofins, do Fust e do Funttel, que são todos tributos da União, e a uniformização das alíquotas de ICMS em 8,5\%, que é um imposto de competência 
dos estados e do Distrito Federal, que, por essa razão, têm autonomia para definir suas próprias alíquotas.

No caso brasileiro, a desoneração tributária tem grande potencial de impacto por duas razões. Em primeiro lugar, porque os tributos representam parcela expressiva do preço do serviço. Com efeito, em 2012, calculamos que o preço mensal médio do serviço era US\$ 40,80', que a alíquota média de ICMS sobre banda larga era $26,67 \%$ e que os tributos correspondiam a 31,82\% do preço médio. Logo, caso a desoneração fosse implantada ainda em 2012 e integralmente abatida da fatura do serviço, o preço seria reduzido para USS 30,60, uma queda de $25 \%$.

A alíquota média de ICMS é calculada ponderando as alíquotas praticadas pelos 26 estados e do Distrito Federal pela participação de cada um deles no número total de assinaturas de banda larga fixa. Para obter o preço mensal médio do serviço de acesso à Internet em banda larga fixa, utilizamos informações relativas à receita tributária dos estados e do Distrito Federal, que, por ser alvo de atenção privilegiada das administrações públicas, são consideradas mais confiáveis e representativas do que fontes alternativas de dados sobre preço desse serviço. 0 cálculo está detalhado no Quadro 2.

\section{Quadro 2. Método de cálculo do preço mensal médio da assinatura do serviço de} acesso à Internet em banda larga-dados agregados dos 26 estados

\section{e do Distrito Federal}

\begin{tabular}{|c|c|c|c|}
\hline $\begin{array}{l}\text { Variáveis e } \\
\text { fórmulas }\end{array}$ & Dados & Fontes & $\begin{array}{c}\text { Referência } \\
\text { temporal }\end{array}$ \\
\hline (a) & Arreca dação de ICMS sobre serviços de comunicação & Confaz ${ }^{(1)}$ & $\operatorname{dez} / 12$ \\
\hline (b) & $\begin{array}{l}\text { Participação do serviço de acesso à Intemet em banda larga } \\
\text { fixa no faturamento do setor de serviços de comunicação }\end{array}$ & $\begin{array}{l}\text { Telebrasil }_{(3)}^{(2)} \\
\text { e Anatel }^{(3)}\end{array}$ & 2012 \\
\hline$(\mathrm{c})=(\mathrm{a})^{*}(\mathrm{~b})$ & $\begin{array}{l}\text { Arrecadação de ICMS sobre serviço de acesso à } \\
\text { Intemet embanda larga fixa }\end{array}$ & $\begin{array}{l}\text { Cálculo } \\
\text { próprio }\end{array}$ & $\operatorname{Dez} / 12$ \\
\hline (d) & $\begin{array}{l}\text { Aliquota média de ICMS sobre o serviço de acesso à Intemet } \\
\text { embanda larga fixa }\end{array}$ & $\begin{array}{l}\text { Cálculo } \\
\text { próprio }\end{array}$ & $\operatorname{Dez} / 12$ \\
\hline (e) & $\begin{array}{l}\mathrm{N}^{\circ} \text { de assinaturas do serviço de acesso à Intemet em banda } \\
\text { larga fixa }\end{array}$ & Anatel $^{(5)}$ & $\operatorname{Dez} / 12$ \\
\hline$(f)=(c) /[(d) *(e)]$ & Preço mensalmédio & $\begin{array}{l}\text { Cálculo } \\
\text { próprio }\end{array}$ & Dez/12 \\
\hline \multicolumn{4}{|c|}{ 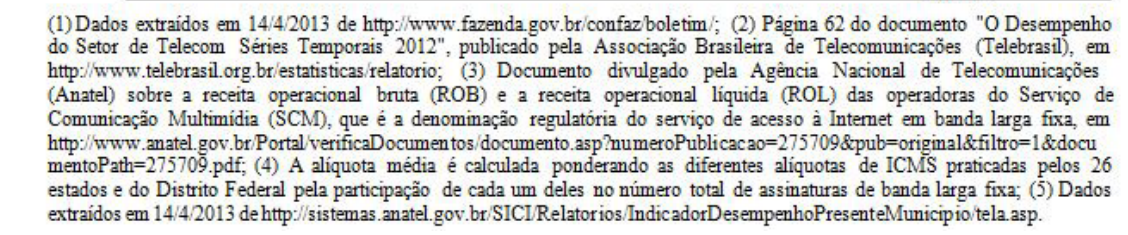 } \\
\hline
\end{tabular}

1 US\$ $1,00=R \$ 2,00$ 
0 peso dos tributos no valor da fatura fica evidente no Quadro 3, abaixo, que apresenta os preços mensais médios (calculados conforme método detalhado no Quadro 2) e os tributos recolhidos de cada assinatura de serviço de acesso à Internet em banda larga fixa de 2010 a 2012. Além disso, apresenta as projeções de preços e de arrecadação de tributos para os anos de 2014, 2018, 2020 e 2022 em dois cenários: sem e com a implantação da proposta de desoneração. A projeção considera que os preços decairão, até 2016, à taxa média anual registrada nos últimos três anos, e que, a partir de 2017, permanecerão constantes. A hipótese subjacente é que os preços deixam de cair porque a competição passa a se concentrar cada vez mais na oferta de acessos de maior velocidade e qualidade com redes de acesso majoritariamente baseadas em fibra óptica, tipicamente mais caros do que os acessos suportados principalmente em pares trançados de cobre e cabos coaxiais.

Quadro 3. Preços mensais médios e tributos recolhidos de cada assinatura do serviço de acesso à Internet em banda larga fixa, sem e com implantação da proposta de desoneração - 2010, 2011, 2012, 2014, 2018, 2020 e 2022

\begin{tabular}{|c|c|c|c|c|c|c|c|c|}
\hline \multirow[b]{2}{*}{$\begin{array}{l}\text { Valores } \\
\text { correntes }\end{array}$} & \multicolumn{4}{|c|}{ Anos realizados } & \multicolumn{4}{|c|}{ Anos projetados } \\
\hline & 2010 & 2011 & 2012 & $\begin{array}{l}\text { Variação } \\
\text { anual média }\end{array}$ & 2014 & 2018 & 2020 & 2022 \\
\hline
\end{tabular}

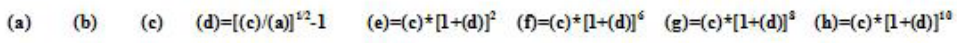

\begin{tabular}{|c|c|c|c|c|c|c|c|c|}
\hline $\begin{array}{l}\text { Preço sem } \\
\text { desoneração }\end{array}$ & 48,37 & 46,86 & 40,80 & $-8,16 \%$ & 34,41 & 29,03 & 29,03 & 29,03 \\
\hline PIS + Cofims $s^{(1)}$ & 1,77 & 1,71 & 1,49 & n.a. & 1,26 & 1,06 & 1,06 & 1,06 \\
\hline Fust+Funttel ${ }^{(2)}$ & 0,51 & 0,49 & 0,43 & n.a. & 0,36 & 0,30 & 0,30 & 0,30 \\
\hline $\operatorname{ICMS}^{(3)}$ & 12,86 & 12,47 & 10,88 & n.a. & 9,18 & 7,74 & 7,74 & 7,74 \\
\hline $\begin{array}{l}\text { Preço com } \\
\text { desoneração }\end{array}$ & n.a. & n.a. & n.a. & n.a. & 25,81 & 21,77 & 21,77 & 21,77 \\
\hline PIS+Cofims $s^{(4)}$ & n.a. & n.a. & n.a. & n.a. & 0,00 & 0,00 & $z 0,00$ & 0,00 \\
\hline Fust+Funttel ${ }^{(s)}$ & n.a. & n.a. & n.a. & n.a. & 0,00 & 0,00 & 0,00 & 0,00 \\
\hline $\mathrm{ICMS}^{(\sigma)}$ & n.a. & n.a. & n.a. & n.a. & 2,19 & 1,85 & 1,85 & 1,85 \\
\hline
\end{tabular}

(1) Aliquota de 3,65\%; (2) Aliquota de 1,5\%; (3) Aliquota média de 26,59\% em 2010, 26,61\% em 2011 e 26,67\% de 2012 em diante; (4) Aliquota zero; (5) Aliquota zero; (6) Aliquota uniforme de $4 \%$.

Em segundo lugar, a desoneração pode ter efeito relevante porque há evidências de que a demanda por serviço de banda larga é bastante sensível a variações de preço. Há uma série de estudos empíricos que estimam valores elevados para a elasticidade-preço da demanda por banda larga fixa no Brasil. Ávila (2008) encontra valores entre -1.00 e -3.36, Guedes, Pasqual, Pitoli e Oliva (2008) obteve -2,00 e Macedo e Carvalho (2010) estimaram valores entre -1,92 e -2,15. Assim, uma eventual redução do preço médio causada pela desoneração tributária redundaria em incremento significativo do número de assinaturas do serviço de banda larga fixa.

Assim, nesta seção, com o intuito de avaliar o impacto potencial da desoneração aqui proposta, estimamos funções de demanda por Internet em banda larga fixa no Brasil. São essas 
funções que possibilitam quantificar a demanda em dois cenários de preço distintos: sem e com a implantação da proposta de desoneração. Para tanto, inspirados no estudo de Macedo e Carvalho (2010), lançamos mão de equações de regressão exponenciais:

$$
D=\mathrm{Se}^{a P}
$$

Em que $P$ é o preço mensal do serviço de acesso à Internet em banda larga fixa, $D$ é a demanda pelo serviço, Sé o nível de saturação da demanda (i.e., a demanda que seria registrada caso o preço do serviço $P$ fosse igual a zero) e $a$ é o fator de amortecimento e um dos componentes da elasticidade-preço da demanda ${ }^{1}$, definida como $e=a P$.

A saturação $S$ e o fator de amortecimento $a$ são parâmetros estimados pelo método de Mínimos Quadrados Ordinários (MOO) a partir de dados discretos de preço $P$ e de demanda $D$. Estes, por sua vez, são obtidos das pesquisas CGI.br $(2011,2012)^{2}$, que indicam a proporção de pessoas que estaria disposta a adquirir acesso à Internet para diferentes níveis preços do serviço, em 2010 e 2011, respectivamente. Os dados são apresentados no Quadro 4.

Quadro 4. Proporção de pessoas que declaram estar dispostas a contratar serviço de acesso à Internet para diferentes níveis de preço mensal do serviço-2010 e 2011.

\begin{tabular}{lccc}
\hline \multicolumn{1}{c}{ Preço } & $\mathbf{2 0 1 0}$ & $\mathbf{2 0 1 1}$ & $\begin{array}{c}\text { Variação } \\
\text { absoluta }\end{array}$ \\
\hline Mais que US\$ 125 & $1 \%$ & $1 \%$ & $0 \%$ \\
Até US\$ 125 & $2 \%$ & $2 \%$ & $0 \%$ \\
Até US\$ 100 & $3 \%$ & $3 \%$ & $0 \%$ \\
Até US\$ 75 & $5 \%$ & $6 \%$ & $1 \%$ \\
Até US\$ 50 & $12 \%$ & $13 \%$ & $1 \%$ \\
Até US\$ 40 & $17 \%$ & $20 \%$ & $3 \%$ \\
Até US\$ 35 & $23 \%$ & $26 \%$ & $3 \%$ \\
Até US\$ 25 & $36 \%$ & $41 \%$ & $5 \%$ \\
Até US\$ 20 & $45 \%$ & $50 \%$ & $5 \%$ \\
Até US\$ 15 & $56 \%$ & $62 \%$ & $6 \%$ \\
Até US\$ 10 & $64 \%$ & $68 \%$ & $4 \%$ \\
Até US\$ 5 & $69 \%$ & $73 \%$ & $4 \%$ \\
Não pagaria nada & $31 \%$ & $27 \%$ & $-4 \%$ \\
\hline
\end{tabular}

Neste estudo, definimos a demanda D por Internet em banda larga como a penetração domiciliar desse serviço (i.e., a proporção de domicílios que possuem esse serviço) e assumimos que este valor é igual à proporção de pessoas que estaria disposta a contratar o serviço de acesso à Internet. Além da indisponibilidade de dados sobre a disposição a pagar por Internet do tipo banda larga fixa, essa igualdade justifica-se por três razões:

1 Assim, quanto maior $a$, mais sensivel é a demanda a variações de preço.

2 Indicador K2: valor máximo declarado para aquisição de acesso à Internet (percentual sobre o total da população com 16 anos ou mais). Vide http://cetic.br/usuarios/tic/2010-total-brasil/rel-aquisicao-02.htm e http://cetic.br/usuarios/ tic/2011-total-brasil/rel-aquisicao-02.htm. 
- As pesquisas do Cetic.br têm caráter domiciliar e seus questionários são submetidos a apenas um morador de cada domicilio;

- De maneira geral, um domicílio é servido por uma única assinatura do serviçode banda larga fixa, que é compartilhado por todos os seus moradores;

- De acordo com essa pesquisa, a grande maioria dos acessos à Internet são do tipo banda larga fixa. De fato, em 2010 e em 2011, mais de 70\% dos acessos domiciliares à Internet era do tipo banda larga fixa.

Abaixo, a Figura 2 apresenta as combinações preço-demanda (as mesmas expostas no Quadro 4) em gráficos de dispersão, as equações de regressão estimadas por M00, o coeficiente de determinação dessas equações e o desenho das curvas por elas geradas para os anos de 2010 e $2011^{3}$. Tanto o traçado das curvas quanto os coeficientes de determinação revelam que o modelo de regressão exponencial se ajusta bem aos dados observados em 2010 e 2011 e, portanto, é adequado para explicar as variações da demanda pelo serviço de Internet em banda larga fixa.

Figura 2. Coordenadas preço-demanda, curva exponencial, equação de regressão e coeficiente de determinação - 2010 e 2011

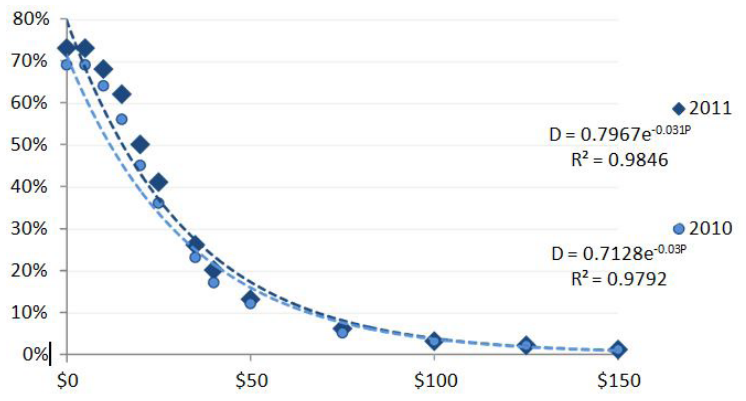

A estimação das funções permite quantificar a demanda para qualquer preço, e não apenas para aqueles considerados pelas pesquisas Cetic.br $(2011$, 2012). Em particular, possibilita mensurar a demanda antes e após a redução de preço resultante da implantação da proposta de desoneração tributária.

Para estimar as curvas de demanda de 2013 a 2022 pelo método aqui adotado é necessário

3 Para estimar o modelo de regressão, a disposição a pagar mais do que US\$125,00 foi arbitrariamente redefinida como a disposição a pagar até US\$ 150,00. Além disso, como os níveis de saturação $S$ estimados não ultrapassaram 1 (i.e., 100\%), optou-se por manter a estimação do modelo de regressão sem impor qualquer restrição sobre os valores de $S$, ainda que os dados mostrem que somente $69 \%$ e $73 \%$ dos domicilios teriam Internet em banda larga mesmo se o serviço fosse gratuito, em 2010 e 2011, respectivamente. Em realidade, os valores dos coeficientes de determinação dos modelos em que $S=0,69$ e $S=0,73$, ou seja, o grau de ajuste dos modelos aos dados, foram inferiores àqueles obtidos pelos modelos irrestritos. 
projetar as coordenadas preço-demanda para cada ano compreendido nesse período de tempo. Isso é feito da seguinte forma:

1. Tomamos como base as coordenadas preço-demanda de 2011 (último ano com dados disponíveis) e adicionamos um múltiplo apropriado da variação anual absoluta da demanda, registrada entre 2010 e 2011, no nível de preço correspondente. Esses dados estão disponiveis para anos anteriores, mas optamos por utilizar a variação anual mais atual por considerarmos que a demanda continuará a aumentar significativamente na próxima década, na medida em que apenas recentemente a população brasileira passou a priorizar a compra de um microcomputador e a aquisição de serviço de acesso à Internet em banda larga fixa.

\section{Introduzimos três restrições lógicas:}

- Imputa-se o valor de 100\% a projeções de demanda projetadas superiores a 100\%;

- Imputa-se o valor de 0\% a projeções de demanda projetadas inferiores a 0\%;

- A demanda em um dado nível de preços deve ser pelo menos igual à demanda no nível de preços imediatamente inferior.

Os Quadros 5 e 6 apresentam as coordenadas preço-demanda observadas em 2010 e 2011 e as projetadas para anos selecionados do período 2013-2022, sem e com a imposição das restrições lógicas (destacadas em itálico), respectivamente.

Quadro 5. Demandas por Internet em banda larga fixa observadas e projetadas, sem imposição de restrições lógicas, para diferentes níveis de preço mensal do serviço 2010, 2011, 2014, 2018, 2020 e 2022

\begin{tabular}{|c|c|c|c|c|c|c|c|}
\hline \multirow{3}{*}{$\begin{array}{l}\text { Preço } \\
\text { mensal }\end{array}$} & \multicolumn{3}{|c|}{ Anos realizados } & \multicolumn{4}{|c|}{ Anos projetados } \\
\hline & \multirow[t]{2}{*}{2010} & 2011 & $\begin{array}{l}\text { Variação } \\
\text { absoluta }\end{array}$ & 2014 & 2018 & 2020 & 2022 \\
\hline & & (b) & $(c)=(b) /(a)-1$ & $(d)=(b)+(c) * 3$ & $(e)=(b)+(c)^{*} 7$ & $(f)=(b)+(c) * 9$ & $(g)=(b)+(c) * 11$ \\
\hline $\begin{array}{l}\text { Mais que } \\
\text { US\$ } 125\end{array}$ & $1 \%$ & $1 \%$ & $0 \%$ & $1 \%$ & $1 \%$ & $1 \%$ & $1 \%$ \\
\hline Até US\$ 125 & $2 \%$ & $2 \%$ & $0 \%$ & $2 \%$ & $2 \%$ & $2 \%$ & $2 \%$ \\
\hline Até US\$ 100 & $3 \%$ & $3 \%$ & $0 \%$ & $3 \%$ & $3 \%$ & $3 \%$ & $3 \%$ \\
\hline Até US\$ 75 & $5 \%$ & $6 \%$ & $1 \%$ & $9 \%$ & $13 \%$ & $15 \%$ & $17 \%$ \\
\hline Até US\$ 50 & $12 \%$ & $13 \%$ & $1 \%$ & $16 \%$ & $20 \%$ & $22 \%$ & $24 \%$ \\
\hline Até US\$ $\$ 0$ & $17 \%$ & $20 \%$ & $3 \%$ & $29 \%$ & $41 \%$ & $47 \%$ & $53 \%$ \\
\hline Até US\$ 35 & $23 \%$ & $26 \%$ & $3 \%$ & $35 \%$ & $47 \%$ & $53 \%$ & $59 \%$ \\
\hline Até US\$ 25 & $36 \%$ & $41 \%$ & $5 \%$ & $56 \%$ & $76 \%$ & $86 \%$ & $96 \%$ \\
\hline Até US\$ 20 & $45 \%$ & $50 \%$ & $5 \%$ & $65 \%$ & $85 \%$ & $95 \%$ & $105 \%$ \\
\hline Até US\$15 & $56 \%$ & $62 \%$ & $6 \%$ & $80 \%$ & $104 \%$ & $116 \%$ & $128 \%$ \\
\hline Até US\$ 10 & $64 \%$ & $68 \%$ & $4 \%$ & $80 \%$ & $96 \%$ & $104 \%$ & $112 \%$ \\
\hline Até US\$ 5 & $69 \%$ & $73 \%$ & $4 \%$ & $85 \%$ & $101 \%$ & $109 \%$ & $117 \%$ \\
\hline $\begin{array}{l}\text { Não pagaria } \\
\text { nada }\end{array}$ & $31 \%$ & $27 \%$ & $-4 \%$ & $15 \%$ & $-1 \%$ & $-9 \%$ & $-17 \%$ \\
\hline
\end{tabular}


Quadro 6. Demandas por Internet em banda larga fixa observadas e projetadas, com imposição de restrições lógicas destacadas em itálico, para diferentes níveis de preço mensal do serviço - 2010, 2011, 2014, 2018, 2020 e 2022.

\begin{tabular}{|c|c|c|c|c|c|c|c|}
\hline \multirow{3}{*}{$\begin{array}{l}\text { Preço } \\
\text { mensal }\end{array}$} & \multicolumn{3}{|c|}{ Anos realizados } & \multicolumn{4}{|c|}{ Anos projetadas } \\
\hline & \multirow[t]{2}{*}{2010} & \multirow{2}{*}{\begin{tabular}{r|}
2011 \\
(b)
\end{tabular}} & \multirow{2}{*}{$\begin{array}{l}\begin{array}{l}\text { Variação } \\
\text { absoluta }\end{array} \\
\text { (c) }=(\text { b) } /(a)-1\end{array}$} & \multirow{2}{*}{$\frac{2014}{(d)=(b)+(c)^{* 3}}$} & \multirow{2}{*}{$\frac{2018}{(e)=(b)+(c)^{* 7}}$} & \multirow{2}{*}{$\frac{2020}{(f)=(b)+(c) * 9}$} & \multirow{2}{*}{$\frac{2022}{(\mathrm{~g})=(\mathrm{b})+(\mathrm{c}) * 11}$} \\
\hline & & & & & & & \\
\hline $\begin{array}{l}\text { Mais que } \\
\text { US\$ } 125\end{array}$ & $1 \%$ & $1 \%$ & $0 \%$ & $1 \%$ & $1 \%$ & $1 \%$ & $1 \%$ \\
\hline AtéUS\$125 & $2 \%$ & $2 \%$ & $0 \%$ & $2 \%$ & $2 \%$ & $2 \%$ & $2 \%$ \\
\hline Até US $\$ 100$ & $3 \%$ & $3 \%$ & $0 \%$ & $3 \%$ & $3 \%$ & $3 \%$ & $3 \%$ \\
\hline Até US\$ 75 & $5 \%$ & $6 \%$ & $1 \%$ & $9 \%$ & $13 \%$ & $15 \%$ & $17 \%$ \\
\hline Até US\$50 & $12 \%$ & $13 \%$ & $1 \%$ & $16 \%$ & $20 \%$ & $22 \%$ & $24 \%$ \\
\hline Até US\$ $\$ 40$ & $17 \%$ & $20 \%$ & $3 \%$ & $29 \%$ & $41 \%$ & $47 \%$ & $53 \%$ \\
\hline Até US $\$ 35$ & $23 \%$ & $26 \%$ & $3 \%$ & $35 \%$ & $47 \%$ & $53 \%$ & $59 \%$ \\
\hline Até US\$25 & $36 \%$ & $41 \%$ & $5 \%$ & $56 \%$ & $76 \%$ & $86 \%$ & $96 \%$ \\
\hline Até US\$20 & $45 \%$ & $50 \%$ & $5 \%$ & $65 \%$ & $85 \%$ & $95 \%$ & $100 \%$ \\
\hline AtéUS\$15 & $56 \%$ & $62 \%$ & $6 \%$ & $80 \%$ & $100 \%$ & $100 \%$ & $100 \%$ \\
\hline Até US\$10 & $64 \%$ & $68 \%$ & $4 \%$ & $80 \%$ & $100 \%$ & $100 \%$ & $100 \%$ \\
\hline Até US\$5 & $69 \%$ & $73 \%$ & $4 \%$ & $85 \%$ & $100 \%$ & $100 \%$ & $100 \%$ \\
\hline $\begin{array}{l}\text { Não pagaria } \\
\text { nada }\end{array}$ & $31 \%$ & $27 \%$ & $-4 \%$ & $15 \%$ & $0 \%$ & $0 \%$ & $0 \%$ \\
\hline
\end{tabular}

Com base nos dados do Quadro 6, construímos as funções de demanda para cada um dos anos do período 2013-2022 utilizando o modelo de regressão exponencial. Na grande maioria dos anos projetados, para não comprometer o ajuste do modelo, optamos por não fixar em 10 nível de saturação e truncamos as funções de forma a garantir que a demanda não superasse 100\% a qualquer nível de preço'.

Os dados projetados para 2014, 2018, 2020 e 2022, as curvas de regressão exponencial, seus coeficientes de determinação e o truncamento das funções de demanda em 100\% são apresentadas na Figura 3.

Figura 3. Coordenadas preço-demanda, equação de regressão, coeficiente de determinação, curva de regressão e truncamento em 100\% - 2014, 2018, 2022 e 2022
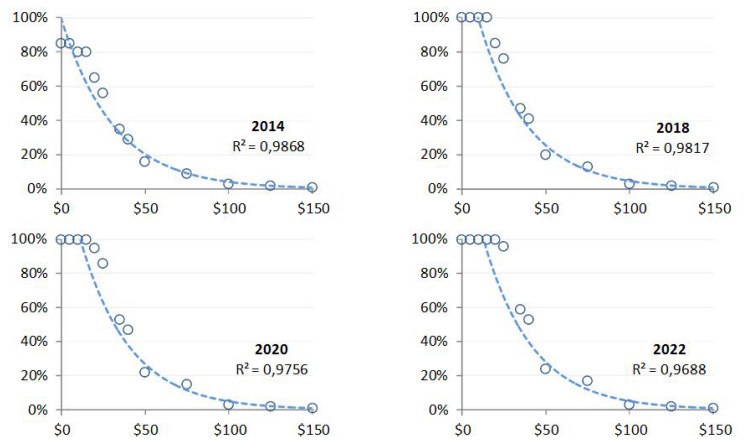

1 Foram truncadas as funções de demanda do ano 2016 em diante. 
0 Quadro 7, a seguir, apresenta os parâmetros das funções de demanda pelo serviço de acesso à Internet em banda larga, além de mostrar os preços mensais médios projetados, sem e com a desoneração tributária, e as demandas correspondentes.

Quadro 7. Parâmetros das funções de demanda, preço mensal médio e demanda estimada do serviço de acesso à Internet em banda larga fixa, sem e com implantação da proposta de desoneração - 2014, 2018, 2020 e 2022.

\begin{tabular}{lcccc}
\hline \multicolumn{1}{c}{ Valores correntes } & $\mathbf{2 0 1 4}$ & $\mathbf{2 0 1 8}$ & $\mathbf{2 0 2 0}$ & $\mathbf{2 0 2 2}$ \\
\hline Nível de saturação(S) & 1,0000 & 1,3916 & 1,4879 & 1,5694 \\
Fator de amortecimento $(\alpha)$ & $-0,0317$ & $-0,0339$ & $-0,0341$ & $-0,0343$ \\
Nível de preço de truncamento ${ }^{(1)}($ US\$ 1,00) & n.a. & 9,74 & 11,64 & 13,15 \\
& & & & \\
Preço sem desoneração (US\$ 1,00) & 34,41 & 29,03 & 29,03 & 29,03 \\
Demanda sem desoneração & $33,6 \%$ & $52,0 \%$ & $55,2 \%$ & $58,0 \%$ \\
& & & & \\
Preço com desoneração (US\$1,00) & 25,81 & 21,77 & 21,77 & 21,77 \\
& & & & \\
Demanda com desoneração & $44,1 \%$ & $66,5 \%$ & $70,7 \%$ & $74,4 \%$ \\
\hline
\end{tabular}

(1) Menor preço para o quala demanda é forçada a igualar-se a 1 (100\%).

A desoneração, se integralmente abatida do valor da fatura mensal do serviço, reduziria em 25\% o preço em todos os anos analisados. Consequentemente, a penetração domiciliar de Internet em banda larga fixa passaria, em 2022, de 58,0\% para $74,4 \%$, o que equivaleria a um aumento de $28 \%$ da demanda por esse serviço. Assim, o modelo desenvolvido nesta seção indica que a elasticidade-preço da demanda por Internet em banda larga fixa para o Brasil é igual a -1,13 no médio prazo².

Resta mensurar a magnitude absoluta do benefício que seria gerado pela desoneração. Isto é, a quantidade de domicílios que passariam a contar com o serviço de acesso à Internet em banda larga fixa como resultado da implantação dessa medida de política pública. Ademais, falta aferir seu custo, medido como a perda de arrecadação tributária (tanto da União quanto dos estados e do Distrito Federal) decorrente da desoneração.

0 Quadro 8, abaixo, evidencia o benefício e o custo da desoneração em anos selecionados do período 2013-2022. Para calcular essas grandezas, projetamos line-

$1(\Delta \mathrm{D} / \mathrm{Q})^{*}\left(\Delta \mathrm{P}^{*} \mathrm{P}\right)=0,28 /-0,25=-1,13$

2 No curtíssimo prazo, isto é, em 2013, a elasticidade-preço calculada é igual a -1,41. 
armente o estoque de domicílios brasileiros para todos os anos do período a partir do levantamento censitário realizado em $2010^{3}$ e das projeções para 2017 e 2022 contidas em EPE (2012).

Quadro 8. Estoque de domicílios e o impacto da desoneração tributária sobre o estoque de domicílios com serviço de acesso à Internet em banda larga fixa e sobre a arrecadação de tributos desse serviço - 2014, 2018, 2020 e 2022.

\begin{tabular}{|c|c|c|c|c|c|}
\hline Valores correntes & 2014 & 2018 & 2020 & 2022 & $\begin{array}{c}\text { Total }(1) \\
(2013-2020)\end{array}$ \\
\hline Estoque total de domicílios (milhões) & 64,6 & 71,6 & 74,5 & 77,5 & 77,5 \\
\hline \multicolumn{6}{|l|}{ Cenário sem desoneração } \\
\hline Estoque de domicílios com banda larga fixa (milhões) & 21,7 & 37,2 & 41,2 & 44,9 & 44,9 \\
\hline Arrecadação de tributos (US\$ bilhões) & 2,81 & 4,07 & 4,50 & 4,91 & 38,19 \\
\hline \multicolumn{6}{|l|}{ Cenário com desoneração } \\
\hline Estoque de domicílios com banda larga fixa (milhões) & 28,5 & 47,6 & 52,7 & 57,6 & 57,6 \\
\hline Arrecadação de tributos (US\$ bilhões) & 0,75 & 1,06 & 1,17 & 1,28 & 9,99 \\
\hline $\begin{array}{l}\text { Benefício da desoneração - adição ao estoque de } \\
\text { domicílios com banda larga fixa (milhões) }\end{array}$ & 6,8 & 10,4 & 11,6 & 12,7 & 12,7 \\
\hline $\begin{array}{l}\text { Custo da desoneração - redução da arrecadação } \\
\text { tributária(US\$ bilhões) }\end{array}$ & 2,1 & 3,0 & 3,3 & 3,6 & 28,2 \\
\hline
\end{tabular}

(1) Igual ao valor de 2022 no caso de variáveis de estoque (quantidade de domicílios); Igual à soma dos valores de 2013 a 2022 no caso das demais variáveis.

Em 2014, na ausência da política de desoneração tributária, o estoque de domicílios com acesso à Internet em banda larga fixa seria igual a 21,7 milhões. Com a desoneração, esse número aumentaria em 6,8 milhões e chegaria a 28,5 milhões. Além disso, a arrecadação tributária, que seria de US\$2,81 bilhões, reduzir-se-ia para US\$ 750 milhões, uma perda de US\$2,1 bilhões.

Portanto, de acordo com o método desenvolvido nesta seção, ao final de 2022, a política de desoneração tributária teria adicionado 12,7 milhões de domicílios ao estoque de domicílios com banda larga fixa e teria custado ao erário o montante de US\$ 28,2 bilhões em valores correntes. Em valores constantes ${ }^{4}$ de 2013, esse montante equivale a US \$ 19,9 bilhões ou R $\$ 40$ bilhões, aproximadamente. 0 Quadro 9 mostra os resultados da função F para essa abordagem.

3 Dados divulgados pelo Instituto Brasileiro de Geografia e Estatistica (IBGE). Podem ser acessados em: http://www. sidra.ibge.gov.br/bda/tabela/listabl.asp?c $=1134 \mathrm{Etz}=\mathrm{cdCto}=7$

4 Considerando uma taxa anual de desconto de $7 \%$. 
Quadro 9. Modelo 1-Desoneração do Serviço: Impacto Projetado

\begin{tabular}{lllll}
\hline & $\mathbf{2 0 1 6}$ & $\mathbf{2 0 1 8}$ & $\mathbf{2 0 2 0}$ & $\mathbf{2 0 2 2}$ \\
\hline Acessos/domicílio & $62 \%$ & $69 \%$ & $74 \%$ & $78 \%$ \\
Velocidade contratada (média) & 15,28 & 24,43 & 31,29 & 36,62 \\
Função Objetivo & 0,8751 & 0,9826 & 1,0812 & 1,1839 \\
\hline
\end{tabular}

Por fim, tendo em vista a possibilidade de uma medida de desoneração ser adotada em conjunto com outras políticas públicas de estímulo à difusão do acesso domiciliar à banda larga fixa, desenhamos uma proposta de desoneração que correspondesse a uma renúncia de arrecadação tributária de $\mathrm{R} \$ 20$ bilhões, metade do montante total de recursos públicos que estariam disponíveis para aplicação em políticas públicas de estímulo à difusão do acesso à Internet em banda larga fixa. Nesse caso, que será detalhado no Modelo 4, as alíquotas de tributos da União continuariam zeradas, mas a alíquota do ICMS seria fixada em 20\%, resultando em um aumento de 6,8 milhões ao estoque de domicílios com banda larga fixa, ao final de 2022.

\section{MODELO 2 - SUBSÍdIO DIRETO AO CONSUMO DO SERVIÇO}

0 modelo de subsídio direto ao consumo do serviço consiste no aporte financeiro mensal ao usuário para contratação do serviço de banda larga. Esse modelo parece ser o indicado para populações de renda extremamente baixa.

Uma restrição política direta dessa proposta é o seu escopo, que necessariamente deve estar definido sobre as famílias de menor renda. Do ponto de vista do resultado para a penetração, essa restrição impede uma alocação ótima dos recursos na medida em que existem domicilios que estariam dispostos a receber um subsídio menor para contratar o serviço, mas que não serão contemplados pela medida por estarem em classes de renda superiores.

Para o cálculo dos resultados, foram utilizadas as mesmas curvas de propensão a pagar detalhadas no Modelo 1, mas desagregadas por classes de renda. Mantendo o foco nas classes de menor renda (famílias com renda mensal inferior a 2 salários mínimos), estimou-se o quanto seria gasto pelo governo para subsidiar o serviço para famílias que estivessem dispostas a pagar ao menos US\$ 2,50 pelo serviço entre os anos de 2013 e 2022. Além disso, para essa proposta considerou-se o custeamento de terminais de acesso para domicílios que não dispunham de microcomputador.

0 Quadro 10 mostra o resultado da função Objetivo com o subsídio direcionado para planos de diferentes preços e velocidades. Para o Plano Básico, Intermediário e Avançado foram adotados os preços mensais de US\$17,5, US\$ 30 e US\$ 50 e as velocidades contratadas iniciais de 1, 4 e 20 Mbps. A redução no preço do Mbps contratado foi 
considerada projetando-se uma evolução anual de $20 \%$ para as velocidades, mantendo-se os preços constantes.

Quadro 10. Modelo 2-Subsídio ao Serviço: Impacto Projetado

\begin{tabular}{lcccc}
\hline & $\mathbf{2 0 1 6}$ & $\mathbf{2 0 1 8}$ & $\mathbf{2 0 2 0}$ & $\mathbf{2 0 2 2}$ \\
\hline Plano 1 - Pacote subsidiado= US\$15,00 & & & & \\
Acessos/domicílio & $55 \%$ & $61 \%$ & $65 \%$ & $69 \%$ \\
Velocidade contratada (média) & 13,09 & 20,77 & 26,86 & 31,31 \\
Função Objetivo & 0,8438 & 0,9489 & 1,0477 & 1,1508 \\
Plano 2 - Pacote subsidiado= US\$ 25,00 & & & & \\
Acessos/domicílio & $50 \%$ & $55 \%$ & $59 \%$ & $62 \%$ \\
Velocidade contratada (média) & 14,59 & 23,50 & 29,97 & 35,61 \\
Função Objetivo & 0,8274 & 0,9320 & 1,0295 & 1,1325 \\
& & & & \\
Plano 3 - Pacote subsidiado= US\$ 50,00 & & & & \\
Acessos/domicílio & $49 \%$ & $55 \%$ & $58 \%$ & $61 \%$ \\
Velocidade contratada (média) & 15,55 & 25,28 & 31,75 & 36,50 \\
Função Objetivo & 0,8276 & 0,9327 & 1,0297 & 1,1316
\end{tabular}

Como pode ser observado, o Plano Básico é o que maximiza a função $F$, ou seja, é recomendável que o subsídio governamental seja direcionado para planos mais baratos, de menor velocidade.

Um dos pontos negativos desse modelo é que subsídios à demanda geram investimentos em infraestrutura apenas de forma indireta. Mais do que isso, para reduzir o custo por família e maximizar a função Objetivo, esse estímulo indireto deve-se dar sobre as tecnologias mais básicas. Assim, não há direcionamento de recursos para tecnologias que assegurem uma maior perpetuidade e que contribuam para a evolução das redes no longo prazo. Ainda, por se tratar de uma garantia de recursos para uma determinada faixa de renda, o aporte governamental pode assumir um caráter de perenidade.

Um dos pontos positivos dessa implementação é o grande impacto na penetração no curto prazo. A questão da perenidade da garantia de recursos pode ser mitigada pela redução gradual do subsídio, por família. Essa redução foi considerada nos cálculos por meio da projeção do aumento da propensão a pagar, conforme explicado no Modelo 1. No entanto, o efeito do aumento relativo da utilidade do serviço em relação aos outros bens e serviços da cesta familiar pode ser ainda maior quando catalisado pelo subsídio para um primeiro contato com o serviço. 


\section{MODELO 3 - INCENTIVOS PARA INVESTIMENTO EM INFRAESTRUTURA}

0 terceiro modelo de repasse governamental é o de financiamento da infraestrutura de rede para regiões de baixa viabilidade econômica no curto e médio prazos. Como explanado na descrição das experiências internacionais, uma das formas de se viabilizar a transferência é por meio de leilões reversos com compromissos de cobertura e de preços.

Uma restrição para esse tipo de implementação é que o investimento governamental deve se dar em tecnologias com algum grau de perpetuidade ("à prova de futuro"). Uma segunda condição, mais intuitiva, é que tais investimentos não poderiam ser direcionados somente a áreas com famílias com maior disposição a pagar pelo serviço.

Nesse sentido, para simular o resultado, foi considerado o repasse governamental para implantação de infraestrutura de rede de acesso $\mathrm{FTH}$, com compromissos de cobertura e de preços para o plano de entrada. Os custos de CapEx e OpEx foram estimados tendo por base a experiência internacional e modelos de negócios para o caso brasileiro. Para a demanda, foram utilizadas as curvas descritas no Modelo 1. Foi obedecida, também, a restrição financeira de US\$20 bilhões para o Valor Presente Líquido - VPL do projeto no período de 2013 a 2030.

Para o preço do serviço, foi estipulado um plano de entrada, com velocidade de 40 Mbps com preço regulado, e pacotes de velocidade mais altas sem regulação de preço. Para o cálculo do Valor Presente Líquido dos fluxos de caixa do modelo de negócio, foram consideradas receitas adicionais com a exploração dos serviços de telefonia e TV por assinatura. A taxa interna de retorno foi fixada em 7\% ao ano.

0 Quadro 10 ilustra o resultado da simulação. Como pode ser observado, ao se reduzir o preço determinado para o plano de entrada, majora-se o número de acessos contratados. No entanto, mantendo-se a restrição financeira, a velocidade média por acesso cai, já que a cobertura que pode ser exigida é menor, reduzindo-se a contratação dos planos não regulados, ou seja, os que podem ser ofertados a preços e velocidades superiores. Em relação aos dois modelos anteriores, essa proposta alcança maiores velocidades médias por acesso em razão do direcionamento do investimento para uma tecnologia de nível superior.

\section{Quadro 11. Modelo 3-Incentivo para investimento em infraestrutura: Impacto Projetado}

\begin{tabular}{|c|c|c|c|c|}
\hline & 2016 & 2018 & 2020 & 2022 \\
\hline \multicolumn{5}{|c|}{ Plano 1 - Preço regulado $=$ US $\$ 10,00$} \\
\hline Acessos/domicilio & $50 \%$ & $55 \%$ & $59 \%$ & $63 \%$ \\
\hline Velocidade contratada (média) & 18,36 & 28,47 & 35,82 & 41,95 \\
\hline Função Objetivo & 0,8348 & 0,9396 & 1,0363 & 1,1428 \\
\hline \multicolumn{5}{|l|}{ Plano 2 - Preço regulado $=$ US $\$ 20,00$} \\
\hline Acessos/domicilio & $47 \%$ & $52 \%$ & $55 \%$ & $58 \%$ \\
\hline Velocidade contratada (média) & 20,57 & 32,28 & 41,26 & 51,59 \\
\hline Função Objetivo & 0,8290 & 0,9320 & 1,0293 & 1,1346 \\
\hline
\end{tabular}


MODELO 4-COMBINAÇÃO DOS MODELOS 1, 2 E 3

0 quarto modelo é uma combinação dos três modelos anteriores, em uma tentativa de extrair os aspectos positivos de cada um e de tentar realizar um melhor ajuste à evolução da função Objetivo (função F).

0 Quadro 12 ilustra o montante de recursos direcionados para cada um dos modelos e os períodos propostos para sua implementação.

Quadro 12. Modelo 4-Solução Combinada: Proposta de Implementação

\begin{tabular}{lccccc}
\hline & $\mathbf{2 0 1 6}$ & $\mathbf{2 0 1 8}$ & $\mathbf{2 0 2 0}$ & $\mathbf{2 0 2 2}$ & $\begin{array}{c}\text { VPL Custo } \\
\text { Governo } \\
\text { (USS bi) }\end{array}$ \\
\hline Modelo 1 - Desoneração do Serviço & $\checkmark$ & $\checkmark$ & $\checkmark$ & $\checkmark$ & 10,00 \\
Modelo 2 - Subsídio ao Serviço & $\checkmark$ & $\checkmark$ & & & 5,00 \\
Modelo 3 - Incentivo para investimento em infraestrutura & & & $\checkmark$ & $\checkmark$ & 5,00 \\
\hline
\end{tabular}

A boa resposta de $\mathrm{F}$ ao modelo de desoneração justifica o maior direcionamento de recursos para essa proposta e a manutenção desse modelo em todo o período. 0 modelo de subsídio é interessante para os primeiros anos porque nesse período a demanda pelo serviço para as classes de menor renda ainda é significativamente baixa. Portanto, nessa implementação há uma redução no desperdício de recursos para o subsídio a famílias que teriam contratado o serviço mesmo sem o programa. Ademais, seu resultado rápido e com impacto significativo na penetração gera maiores impactos em $\mathrm{F}$ nos primeiros anos, quando o peso relativo de $a$ ainda é maior que o de $v$ (Figura 1).

Empregar o Modelo 3 apenas nos últimos cinco anos e de forma combinada com as outras propostas otimiza significativamente o resultado do programa em relação à proposta isolada descrita no Modelo 3. Primeiramente, os ganhos de velocidade média do modelo geram maior impacto na função $F$ nos últimos anos, em razão da majoração gradual do parâmetro $v$ da função Objetivo ao longo tempo. Depois, há uma redução significativa nos gastos do governo devido à evolução da propensão a pagar nos últimos anos e à redução no CapEx para construção da rede de acesso FTTH. Finalmente, utilizar o modelo de forma combinada com as duas outras propostas permite flexibilizar a restrição política de investimento em todas as regiões do país. Como já existirão propostas direcionadas para as classes de menor renda (subsídio ao serviço) e para a população como um todo (desoneração), os investimentos em infraestrutura podem se dar inicialmente em municípios de maior densidade populacional, o que reduz o custo da cobertura, e para regiões com maior disposição a pagar pelo serviço.

0 Quadro 13 mostra o resultado para essa proposta. 
Quadro 13. Modelo 4-Solução Combinada: Impacto Projetado

\begin{tabular}{lcccc}
\hline & $\mathbf{2 0 1 6}$ & $\mathbf{2 0 1 8}$ & $\mathbf{2 0 2 0}$ & $\mathbf{2 0 2 2}$ \\
\hline Acessos/domicilio & $63 \%$ & $70 \%$ & $69 \%$ & $70 \%$ \\
Velocidade contratada(média) & 15,20 & 24,35 & 44,28 & 65,52 \\
Função Objetivo & 0,8775 & 0,9840 & 1,0808 & 1,1853 \\
\hline
\end{tabular}

\section{Conclusão}

0 estudo explorou modelos recentes que vêm sendo adotados para expansão do serviço de acesso à banda larga. Dentre os modelos descritos três foram analisados sob o ponto de vista de seu impacto nos índices de penetração e de velocidade média: redução nos tributos sobre o serviço, subsídio direto para o consumo do serviço e incentivo para investimento em infraestrutura. Para estimar o impacto de cada uma das propostas, foi construida uma função Objetivo que teve por base o ICT Development Index da UIT. 0 modelo de redução de tributos gerou impactos bastante significativos na penetração do serviço, já que o rebaixamento geral do nivel de preços permite que famílias com maior disposição a pagar passem a contratar o serviço. 0 modelo de subsídio apresentou um bom impacto de curto prazo na penetração, mas peca pelo direcionamento do investimento somente para tecnologias mais básicas, pelo desperdício crescente com o financiamento de famílias que já contratariam o serviço e pela restrição política de subsidiar famílias com menor propensão a pagar. 0 modelo de incentivo a investimentos em infraestrutura gerou boa resposta no parâmetro de velocidade, mas baixo impacto no aumento do número de acessos contratados. Ao final, foi proposto um quarto modelo, que tentou extrair os pontos positivos de cada uma das modelagens iniciais e que se alinhasse melhor a variações dos parâmetros $a$ e $v$ de F. Esse modelo foi o que maximizou a função Objetivo para a maior parcela do período estudado.

\section{Referências}

Ávila, F. (2008) Banda larga no Brasil: uma análise da elasticidade preço-demanda com base em microdados, Monografia de graduação do curso de Economia, Orientadora: Maria Eduarda Tannuri-Pianto, Universidade de Brasilia (UnB).

Calvo, A. G. (2012) Universal Service Policies in the Context of National Broadband Plans, OECD Digital Economy Papers, No. 203, OECD Publishing. Disponível em: http://dx.doi. org/10.1787/5k94gz19flq4-en.

CEPAL. (2012) Estado de la Banda Ancha en América Latina y el Caribe, 2012: Informe del 
Observatorio Regional de Banda Ancha (ORBA). Disponivel em: http://www.cepal.org/ Socinfo.

COMISSÃO EUROPEIA. (2012) The socio-economic impact of bandwidth. Final Report. Elaborado por Analysys Mason Ltd e Tech4i2 Ltd. SMART 2010/0033. Disponível em: http://ec.europa.eu/digital-agenda/en/news/study-socio-economic-impact-bandwidth-smart-20100033.

COMISSÃO DE COMÉRCIO. (2012) High speed broadband services demand side study, New Zealand. Disponivel em:

http://www.comcom.govt.nz/high-speed-broadband-services-demand-side-study

CGI.br. (2011) TIC Domicílios e Empresas 2010: Pesquisa sobre o Uso das Tecnologias de Informação e Comunicação no Brasil, Comitê Gestor da Internet no Brasil (CGI.br), São Paulo. Disponivel em: http://op.ceptro.br/cgi-bin/cetic/tic-domicilios-e-empresas-2010.pdf. . (2012) TIC Domicilios e Empresas 2011: Pesquisa sobre o Uso das Tecnologias de Informação e Comunicação no Brasil, Comitê Gestor da Internet no Brasil (CGI.br), São Paulo. Disponível em: http://op.ceptro.br/cgi-bin/cetic/tic-domicilios-e-empresas-2011.pdf.

EPE. (2012) Projeção da demanda de energia elétrica para os próximos 10 anos: 2013-2022, Série Estudos da Demanda, Empresa de Pesquisa Energética (EPE), Rio de Janeiro. Disponivel em: http://www.epe.gov.br/mercado/Documents/S\%C3\%A9rie\%20Estudos\%20 de\%20Energia/20130117_1.pdf.

Galperín, H., Mariscal J. e Viecens, M. F. (2012) Análisis de los Planes Nacionales de Banda Ancha en América Latina.

ACORN-REDECOM 2012. Valparaiso, Chile. Disponivel em: http://www.acorn-redecom.org/ papers/proceedings2012/013Galperin_Espanol.pdf

Guedes, E. M., Pasqual, D. de, Pitoli, A. e Oliva, B. (2008) Avaliação dos impactos da cisão das operações de STFC e SCM em empresas distintas. Nota Técnica, Tendências Consultoria Integrada, São Paulo. Disponível em: http://www.anatel.gov.br/Portal/verificaDocumentos/documento.asp?numeroPublicacao $=216576$ ctassuntoPublicacao $=$ Pr ocesso $\% 20$ altera\%E7\%E30\%20do\%20PG0\&tcaminhoRel=null\&tiltro=1\&tocumentoPath=216576. pdf (págs. 16 a 32).

Hardy, A. (1980) The role of the telephone in economic development. Telecommunications Policy 4, 278-286.

Jordán, V., Galperin H. e Peres, W. (2010) Acelerando la revolución digital: banda ancha para América Latina y el Caribe, CEPAL-DIRSI, Santiago de Chile. Disponível em: http:// www.eclac.cl/publicaciones/xml/7/41727/LCR.2167.pdf

Koutrompis, P. (2009) The economic impact of broadband on growth: a simultaneous approach, Telecommunications Policy, 33, 471-485.

ITU. (2012) Measuring the Information Society 2012, Geneva, Switzerland. Disponivel em: http://www.itu.int/ITU-D/ict/publications/idi/material/2012/MIS2012_without_Annex_4.pdf

Macedo, H. R. e Carvalho, A. X. Y. (2010) Aumento da penetração do serviço de acesso à 
Internet em banda larga e seu possível impacto econômico: análise através de sistema de equações simultâneas de oferta e demanda, Série Textos para Discussão, Instituto de Pesquisa Econômica Aplicada (Ipea), Rio de Janeiro. Disponivel em: http://www.ipea. gov.br/portal/images/stories/PDFs/TDs/td_1495.pdf.

Proyecto Todo Chile Conectado. (2010) Proyecto Bicentenario "Red de Internet Rural: Todo Chile Conectado". Ministerio de Transportes y Telecomunicaciones, Subsecretaría de Telecomunicaciones - DGFDT. Disponível em: http://www.subtel.gob.cl/images/stories/ articles/subtel/asocfile/ppt_bicentenario_fdt_red_internet_rural.pdf

Qiang, C. e Rossotto, C. (2009) Economic impacts of broadband. Information and Communications for Development. Banco Mundial. Disponivel em: http://siteresources.worldbank. org/EXTIC4D/Resources/IC4D_Broadband_35_50.pdf.

Vive Digital. (2011) Ministerio de Tecnologías de la Información y Comunicaciones, República de Colombia. Disponível em: http://www.vivedigital.gov.co/files/Vivo_Vive_Digital.pdf. WEF. (2013) The Global Information Technology Report 2013, Geneva, Switzerland. Disponivel em: http://www3.weforum.org/docs/WEF_GITR_Report_2013.pdf 